Tobias Kortus, Thilo Krüger*, Gabriele Gühring and Kornelius Lente

\title{
Automated Robust Interpretation of Intraoperative Electrophysiological Signals - A Bayesian Deep Learning Approach
}

\begin{abstract}
Intraoperative neurophysiological monitoring (IONM) is an essential tool during numerous surgical interventions to assess and monitor the functional integrity of neural structures at risk. A reliable signal interpretation is of importance to support medical staff by reducing manual evaluation. Deep learning (DL) techniques proved to be a robust tool for the analysis of neurophysiological data. The large amount of required manually labeled data as well as the lack of interpretability of the results however often limit the use of DL in medical scenarios. A possible way to tackle these obstacles is the utilization of Bayesian deep learning (BDL) methods. The modelling of uncertainties in the network parameters and the thereby possible quantification of predictive uncertainties allows both the identification of potential erroneous predictions as well as the targeted selection of informative signals in the context of active learning. To evaluate the applicability of BDL for the analysis of electrophysiological data as well as to increase the training efficiency by active learning, we implemented a multi-task Bayesian Convolutional Neural Network (BCNN) for the simultaneous classification of action potentials and the assessment of relevant signal characteristics (latency, maximum, minimum). We compare the results for electromyographical signals (EMG), containing in total approximately twelve thousand signals from 34 patients, with both a traditional non-Bayesian single-task and multi-task CNN. For all models, including the BCNN, we could achieve similar performances with detection rates over $97 \%$ accuracy. Further, we could improve training efficiency of the BCNN using pool-based active learning and therefore significantly reduce the required amount of manual labeling. The evaluated predictive uncertainties of the $\mathrm{BCNN}$ prove useful both for the efficient selection of informative signals in the context of
\end{abstract}

\footnotetext{
*Corresponding author: Thilo Krüger: inomed Medizintechnik GmbH, Im Hausgrün 29, 79312 Emmendingen, Germany, e-mail: t.krueger@inomed.com

Tobias Kortus, Kornelius Lente: inomed Medizintechnik GmbH Emmendingen, Germany

Gabriele Gühring: Hochschule Esslingen, Esslingen, Germany
}

active learning as well as the interpretation of the predictive posterior distribution and therefore trustworthiness of the classifications.

Keywords: electromyography, intraoperative neuromonitoring, Bayesian deep learning, convolutional neural networks, pool-based active learning

https://doi.org/10.1515/cdbme-2021-2018

\section{Introduction}

Intraoperative neurophysiological monitoring (IONM) is a widely used technique for intraoperatively monitoring intact neural structures to avoid nerve damages and indicate possible damages at an early state in order to avoid postoperative damages or loss of functionality [1]. This includes the monitoring of several important physiological signal parameters of the derived signals indicating the current functional state of nerves at risks. A lot of effort has been put in developing suitable algorithms for automatically evaluating the signals both using traditional and deep learning algorithms. Most approaches however lack the ability to interpret internal (epistemic) model uncertainties making the trustworthiness of the predictive outputs not feasible. This becomes in particular critical for complex signal types not represented in the distribution of the training and evaluation datasets. In those cases, possible erroneous extrapolations of the classification results are often not handled in a sufficient way leading to unjustifiably overconfident predictions (ref. figure 1).

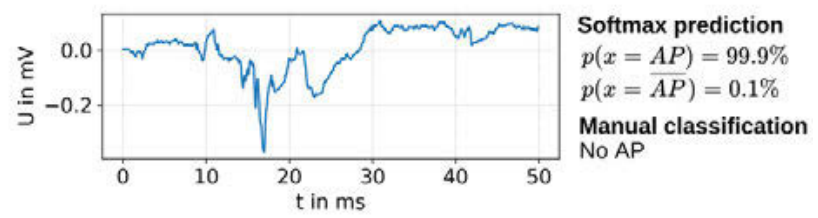

Figure 1: Overconfident erroneous false prediction of an electrophysiological signal produced by the t-LeNet architecture described in 2.1 
Bayesian Machine Learning and in particular Bayesian Deep Learning allows us to efficiently model those uncertainties in an efficient way, leveraging theoretical properties of Bayesian statistics [2].

\section{Methods}

\subsection{Network Architectures}

We evaluate the eligibility of Bayesian Deep Learning methods for electrophysical signals in three incremental steps in order to detect possible negative influences induced by the supportive methodologies as soon as possible. We therefore introduce a basic CNN model adapted from the t-LeNet architecture by Guennec et al. [3], containing two convolutional and two fully connected layers with ReLu activation, for the classification of the raw inputs signals into either action potentials (AP) or non-action potentials (no AP). We extend this model by a second output branch containing two hidden layers with sigmoid activation (t-LeNet-MT) for the assessment of relevant signal characteristics including latency of the response signal as well as signal maxima and minima. The modified training routine required for the simultaneous training of the joint multi-task network is further described in 2.2. For the Bayesian model (t-LeNet-MT-MCD) we use Monte Carlo Dropout Sampling as proposed by Gal et al. [4] as a simple yet efficient Bayesian approximation for inferring an approximate of the true posterior distribution without requiring a modification of neither the model structure nor the training routine. We therefore add dropout layers, with a deactivation rate of 0.5 , applied both during training and test time, after every network layer containing trainable weights. We evaluate the predictive posterior distribution for MonteCarlo Dropout using 50 randomly sampled dropout configurations.

\subsection{Network Training}

For the simultaneous training of both multi-task CNN's network branches, to obtain optimal network parameters maximizing the predictive performance of each task, an adaption of the training routine is required. Instead of a single loss function a joint loss function containing a weighted sum of the individual tasks (classification and regression) is optimized. To avoid the manual fine tuning of the individual weighting of the loss functions we use an automatic weighting based on the homoscedastic (task) uncertainties obtained for both tasks as proposed by Kendall et al. [5].

\subsection{Uncertainty Quantification}

To numerically quantify the uncertainties obtained by the predictive posterior distribution we use a calibrated uncertainty measure over the variance of the Monte-Carlo samples as described in Gal et al. [4]. We therefore model a Gaussian distribution, parametrized by a mean $(\mu)$ and standard deviation $(\sigma)$ over the variance obtained from predictive posterior distributions of the training set. In order to scale this approach efficiently to large datasets we estimate the mentioned parameters incrementally using Welford's algorithm [6]. We obtain the final, scaled uncertainty estimates for new predictions by calculating the cumulative probability for new incoming data.

\subsection{Pool-Based Active Learning}

Given the normally homogeneous signal characteristics obtained during usual surgical interventions, the distribution over possible signals does not match the i.i.d assumption of stochastic optimization algorithms used for network training. Instead, a small number of signal types is strongly overrepresented making the training of neural networks for a robust and error-resistant operation inefficient. Thus, either a large volume of labeled training data, containing to a large extend uninformative signals, or a manual pre-selection of the most informative signals is required. Pool-based active learning provides an automatic mechanism to select the most informative signals from an unlabeled data pool iteratively by maximizing an arbitrary form of acquisition function (i.e. in (4)) based on the given model. We use a batchwise selection of informative samples $\left\{x_{1}^{*}, \ldots, x_{b}^{*}\right\}$ given a model $p\left(\omega, \mathcal{D}_{\text {train }}\right)$ that maximizes the defined acquisition function $a(\cdot)$ :

$$
\left\{x_{1}^{*}, \ldots, x_{n}^{*}\right\}=\arg \max a\left(\left\{x_{1}, \ldots, x_{n}\right\}, p\left(\omega, \mathcal{D}_{\text {train }}\right)\right)
$$

We evaluate the performance of active learning for electrophysiological signals based on two information theoretical acquisition functions reducing the total uncertainty of the model predictions. Our first acquisition estimates the total entropy over all predictions of a batch size $n$ :

$$
a_{E n t}=\sum_{i=1}^{n} \mathbb{H}\left(y_{i} \mid x_{i}, \mathcal{D}_{\text {train }}\right)
$$

As a second metric we evaluate BatchBALD as proposed by Kirsch et al. [7]. This approach formulates the acquisition of informative data as an optimization of the mutual information between the model parameters $\boldsymbol{\omega}$ and the predicted model output $y$. In contrast to the acquisition score based on the total 
Entropy which is evaluated for each signal separately, this approach optimizes a joint score over a batch of signals.

$$
a_{\text {BatchBALD }}=\mathbb{I}\left(y_{1: n} ; \boldsymbol{\omega} \mid x_{1: n}, \mathcal{D}_{\text {train }}\right)
$$

\subsection{Model Evaluation}

The data used throughout this paper, provided by inomed Medizintechnik $\mathrm{GmbH}$, was collected during multiple thyroid surgeries from 34 patients in total. Each of the individual signals contains a single EMG channel recorded at $20 \mathrm{kHz}$ with $50 \mathrm{~ms}$ each. In order to balance the overall class distribution (AP and no AP) we perform an under sampling of the individual patients to a total of 200 signals per patient per class. We use the Adam [8] optimizer for training the network with different training parameters determined for each individual architecture by a hyperparameter optimization using Optuna [9]. Further we use a five-fold cross validation to obtain independent and balanced results.

\section{Results}

\subsection{Predictive Performance}

To verify the impact and quality of Bayesian methods for the analysis of electrophysiological signals we evaluate the predictive performances for all network architectures as described in 2.5. All results of the evaluation are shown in tables 1 and 2. For the classification of action potentials we could achieve accuracies above the $97 \%$ accuracy mark for all network architectures. The best results were obtained by the tLeNet-MT architecture with $97.2 \%$. For the Bayesian approach we could determine a slightly decrease in accuracy by approximately $0.5 \%$ compared to the previous architecture.

Table 1: Obtained predictive performance (accuracy, precision and recall) and mean squared error (MSE) obtained for classification of action potentials and assessment of signal characteristics for the network architectures described in section 2.1.

\begin{tabular}{lllll}
\hline Model & Accuracy & Precision & Recall & MSE \\
\hline t-LeNet & $97.2 \%$ & $97.4 \%$ & $96.3 \%$ & - \\
t-LeNet-MT & $97.6 \%$ & $97.7 \%$ & $97.6 \%$ & $0.0010 \mp 0.0003$ \\
t-LeNet-MT- & $97.2 \%$ & $97.6 \%$ & $96.4 \%$ & $0.0020 \mp 0.0003$ \\
MCD & & & & \\
\hline
\end{tabular}

For the identification of signal latency, maximum and minimum we could determine significant better results for the multi-task architecture without Monte-Carlo Dropout Sampling (ref. table 2). Further we show that additional fine tuning of the weights of the regression branch further increases the quality of the predictions.

\subsection{Uncertainty Quantification}

Evaluating the predictions of the Bayesian CNN for multiple dropout configurations sampled from the learned posterior distribution allows us to infer a predictive distribution over the network outputs. As depicted in figure 2 this allows to visually determine uncertain predictions. This assessment however is highly subjective and requires further manual evaluation.
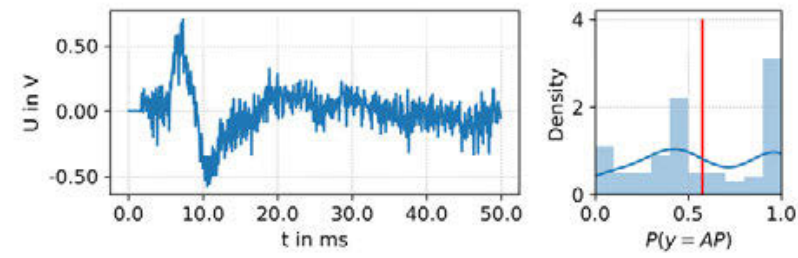

Figure 2: Predictive posterior distribution of an uncertain classification of an EMG signal obtained by the t-LeNet-MTMCD architecture.

We therefore evaluate the calibrated uncertainty measure described in 2.3. The optimization of the required parameters is performed on the uncertainty estimates of the training dataset after the initial training of the Bayesian model. To evaluate the quality of the metrics ability to capture the relationship between uncertainty values and correctness of classifications we calculate the uncertainty values for all data samples from the training dataset. The corresponding distributions for the classification categories (true positives (TP), true negatives (TN), false positives (FP) and false negatives $(\mathrm{FN})$ ) are depicted in figure 3.

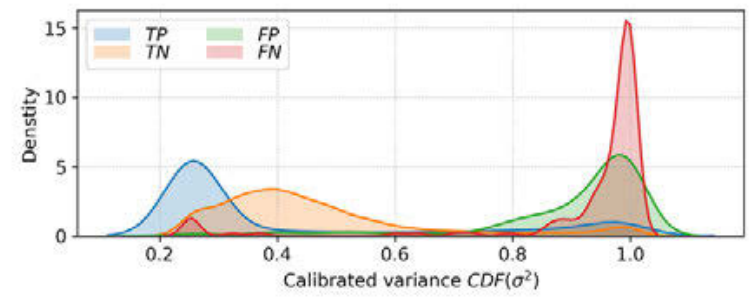

Figure 3: Distribution of the obtained calibrated uncertainty estimates for the categories TP, TN, FP, FN.

Examining the distributions of the true and false classifications, we find a clear separation making the distinction between true and false classifications based on the predictive uncertainty possible. However, there are multiple 
areas in which true and false classifications overlap. Thus, an unambiguous identification of false classifications is not possible.

\subsection{Active Learning}

As a final comparison between active and passive learning for training the models we evaluate the acquisition functions for pool-based active learning, described in section 2.4. We therefore monitor the accuracy of a single-task variant of the Bayesian CNN over one hundred acquisition steps with a batch size of sixteen signals each. In order to obtain well-founded results, we repeated the procedure five times in order to reduce random effects.As depicted in figure 4 we can see a significant

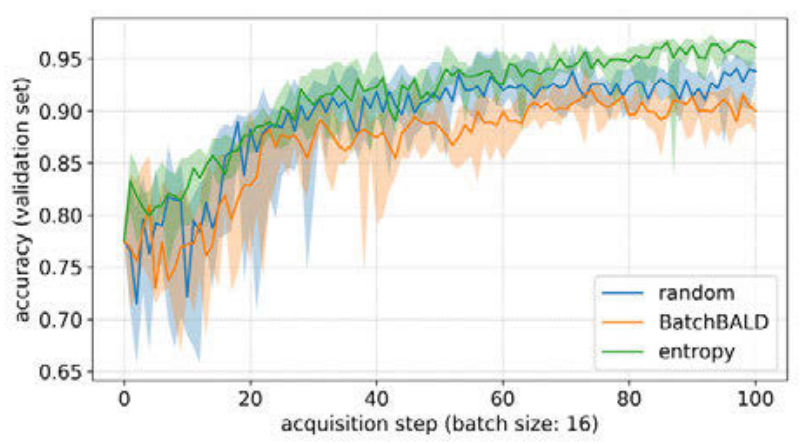

Figure 4: Training accuracies of the t-LeNet network architecture determined for various acquisition functions.

improvement in accuracy when using the acquisition function based on the entropy of the prediction. However, the BatchBALD acquisition did not yield any improvement, instead we report a steady deterioration of the results.

\section{Discussion}

In this work we show that the utilization of Bayesian CNNs is well suited for the robust analysis of electrophysiological signals. We prove that an increased source of confidence can be introduced using the predictive uncertainty as an indicator of trustworthiness of the predictions without losing predictive accuracy. However, for the more complex regression task we notice a significant deterioration in accuracy because of the thinning of the network architecture during inference due to the application of Monte Carlo sampling. This phenomenon however can most likely be further reduced by additional training. Furthermore, the evaluated pool-based active learning, utilizing the estimated predictive uncertainty as a measure of the informative of the analysed signals, proves to be a valuable extension to the training pipeline of the deep learning approach. An existing drawback of the currently used algorithm for the Bayesian interpretation of the used neural network is the increased complexity during inference due to the Mote-Carlo estimation of the predictive posterior distribution. Focus of future work will therefore include the analysis of sampling-free estimation of uncertainties as well as the enlargement of the signal dataset to further improve the performance of the model.

\section{Author Statement}

Research funding: The author state no funding involved. Conflict of interest: Authors state no conflict of interest. Informed consent: Informed consent has been obtained from all individuals included in this study. Ethical approval: The research related to human use complies with all the relevant national regulations, institutional policies and was performed in accordance with the tenets of the Helsinki Declaration, and has been approved by the authors' institutional review board or equivalent committee.

\section{References}

[1] Zentner, J., \& Wegner, C. (2019). Intraoperatives Neuromonitoring: Grundlagen, Möglichkeiten, Grenzen (1st ed.). MWV Medizinisch Wissenschaftliche Verlagsgesellschaft.

[2] Ghahramani, Z. (2015). Probabilistic machine learning and artificial intelligence. Nature, 521(7553), 452-459.

[3] Le Guennec, A., Malinowski, S., \& Tavenard, R. (2016). Data Augmentation for Time Series Classification using Convolutional Neural Networks. ECML/PKDD Workshop on Advanced Analytics and Learning on Temporal Data,

[4] Gal, Y., \& Ghahramani, Z. (2016). Dropout as a Bayesian approximation: Representing model uncertainty in deep learning. 33rd International Conference on Machine Learning, ICML 2016, 3, 1651-1660.

[5] Kendall, A., Gal, Y., \& Cipolla, R. (2018). Multi-task Learning Using Uncertainty to Weigh Losses for Scene Geometry and Semantics. Proceedings of the IEEE Computer Society Conference on Computer Vision and Pattern Recognition, 7482-7491.

[6] Welford, B. P. (1962). Note on a Method for Calculating Corrected Sums of Squares and Products. Technometrics, 4(3), 419-420.

[7] Kirsch, A., van Amersfoort, J., \& Gal, Y. (2019). BatchBALD: Efficient and Diverse Batch Acquisition for Deep Bayesian Active Learning. Advances in Neural Information Processing Systems (Vol. 32).

[8] Kingma, D. P., \& Ba, J. L. (2015). Adam: A method for stochastic optimization. 3rd International Conference on Learning Representations, ICLR 2015 - Conference Track Proceedings, 1-15.

[9] Takuya Akiba, Shotaro Sano, Toshihiko Yanase, Takeru Ohta, and Masanori Koyama (2019). Optuna: A Nextgeneration Hyperparameter Optimization Framework. In KDD 